\title{
Intrahepatic Cholestasis in Pregnancy: Increased Surveillance and the Role of Bile Acids in a Patient with History of Fetal Demise
}

\author{
Noah Ryan Begley* and Abdalyz Frias Beltran \\ School of Medicine, Universidad Autónoma de Guadalajara, USA
}

*Corresponding author: Noah Ryan Begley, School of Medicine, Universidad Autónoma de Guadalajara, Phoenix, Arizona, USA, Tel: 480-428-9950

\begin{abstract}
Intrahepatic cholestasis of pregnancy (ICP) is a known risk for fetal demise and premature delivery. We describe the case of a third-trimester patient with ICP that would have gone unnoticed based on laboratory reporting. Symptoms presented two weeks after laboratory values consistent with ICP were obtained, and serial labs fell below the threshold despite persistent symptoms. The diagnosis was further complicated when the types of bile acids and the ranges for normal varied between facilities. We emphasize the importance of assessing lab values objectively and investigating the causes of pregnant patients' itching.
\end{abstract}

\section{Keywords}

Cholestasis, Pregnancy, Obstetrics, Bile acids, Intrahepatic, Gastroenterology, Biliary

\section{Introduction}

Nearly a quarter of pregnant patients experience pruritus significant enough to report to their provider. Most cases are dermatological in origin and present with some evidence. Intrahepatic cholestasis of pregnancy (ICP) is one cause of pruritus that presents without a rash and has more severe consequences than other causes of itching. The risk is higher for Latin females who have an incidence of up to $15 \%$ versus the general obstetric population $0.3-0.5 \%$ [1]. The typical presentation of ICP is second or third trimester and includes pruritus in the palms and soles that worsens at night. Other symptoms of ICP include abdominal pain, nausea, poor appetite, and subclinical steatorrhea [2].
The causes of ICP are poorly understood, but are believed to be a combination of genetic, hormonal, and environmental factors. Mutations in phospholipid and bile salt export pumps found in the liver and placentas of ICP patients are believed to be factors [3-5]. An increased amount of the smooth muscle relaxant estrogen during pregnancy is another known contributor to stasis and the buildup of bile acids. Increased progesterones conjugation in the hepatic export system leads to inflammation and could play an additional role.

During pregnancy, serum bile acids within the fetus are slightly higher than those in the maternal circulation where they are ultimately transferred for excretion [6]. In ICP, the fetomaternal concentration gradient is reversed and is known to increase risk of fetal complications such as stillbirth, spontaneous preterm birth, and vasospasms leading to abnormal ECG findings [7]. In particular, it has been shown that fetal cardiomyocytes have increased susceptibility to and incidence of tachycardia, bradycardia, atrial flutter, and supraventricular tachycardia when exposed to elevated bile acids [8].

TBA values $>10-20$ umol/L (varies with lab) during second and third trimesters is considered diagnostic [9]. Evaluation includes history and physical exam and complete blood count (CBC), liver function tests (LFT), TBA, and weekly fetal biophysical profile (BPP). The debate on whether TBA should be collected fasting or postprandial has remained. LFTs are elevated in

Citation: Begley NR, Beltran AF (2021) Intrahepatic Cholestasis in Pregnancy: Increased Surveillance and the Role of Bile Acids in a Patient with History of Fetal Demise. Obstet Gynecol Cases Rev 8:212. doi.org/10.23937/2377-9004/1410212

Accepted: October 09, 2021: Published: October 11, 2021

Copyright: (c) 2021 Begley NR, et al. This is an open-access article distributed under the terms of the Creative Commons Attribution License, which permits unrestricted use, distribution, and reproduction in any medium, provided the original author and source are credited. 
upto $60 \%$ of cases although their role in management is nonexistent. More common causes of itching such as dermatitis, and more severe causes such as HELLP (hemolysis, elevated liver enzymes, and low platelet count) syndrome must also be ruled out.

The use of ursodeoxycholic acid (UDCA) has demonstrated efficacy in the treatment of symptomatic ICP cases and, reducing the likelihood of neonatal cardiac abnormalities [7]. Should bile acids reach or exceed 100 umol/L, and symptoms are refractory to treatment or the patient has a history of intrauterine demise due to ICP delivery starting at $360 / 7$ has shown to reduce the risks of stillbirth (Ovadia). For those with TBA between 40-99 umo1/L or persistent symptoms delivery between 36 0/7 - 39 0/7 is recommended [10]. Postpartum bile acids should be collected at 4-6 weeks to reevaluate a patient's status. If hepatobiliary dysfunction is persistent by laboratory standards a referral to gastroenterology is warranted (Pillarisetty). Succeeding pregnancies should be monitored for ICP given there is a $60-90 \%$ chance of recurrence [11].

\section{Case Presentation}

A 30-year-old G5P3 female with a history of unexplained fetal demise presented to the clinic at 37 +1 having just been discharged from the hospital's obstetrics department. The patient had an uneventful pregnancy to that point but four days earlier presented to the hospital obstetrics floor complaining of wholebody itching with pronounced pruritus on the hands and feet. In the office, the physical exam was unremarkable except for scratch marks on the lateral aspect of one leg.

Fetal heart tones were within normal limits (WNL) and she reported fetal movements daily with no abdominal pain or vaginal bleeding. Urinalysis was unremarkable for signs of infection or preeclampsia and the patient was normotensive. A review of her hospital labs showed a bile TBA of $7 \mathrm{umol} / \mathrm{L}$ and included fractionated bile acids (FBA). A routine complete metabolic panel (CMP) obtained 3 weeks earlier showed a TBA consistent with ICP at I1 umol/L although this was not considered elevated by that lab's reference ranges and the patient had no symptoms. A repeat CMP with TBA was taken in the clinic and showed that TBA had decreased to 4 umo1/L. Although values fell below the diagnostic threshold the patient's itching worsened and was consistent with ICP. The following evening she presented to the hospital with worsening of her itching. Given earlier lab confirmation of ICP, refractory symptoms, and history of fetal demise a decision was made with the patient to undergo induction at 373/7. A healthy full-term baby was delivered without incident. At a 3-week follow-up, the patient reported cessation of pruritus and a thriving newborn.

\section{Discussion}

In this case, we found that different labs measured different acid types and had different thresholds for 'elevated' [12]. Further comparison with labs across the country revealed that the values changed even more dramatically with distance [13]. Manzotti, et a1. found that neither value was significantly sensitive or specific for the diagnosis of ICP or the management of symptoms [14]. Perinatology researchers have suggested that neutrophil to leukocyte ratio could serve as a more specific measurement of disease severity given the role inflammation plays in ICP [15]. A more sensitive or specific sign would be clinically helpful, however, we agree that future efforts should focus on establishing consistency for reference ranges between laboratories and the significance of fractionated bile acids. Moreover, it is not clear when the benefits of UDCA are maximized and should be evaluated on a chronometric scale to better understand the benefits and to reduce the iatrogenic effects of pharmacological intervention and preterm delivery.

Obstetricians are in a position to survey risk factors unique to their patients such as those who have had a cholestatic response to oral contraceptives [16] and have the benefit of more frequent visits. At-risk populations should not exclude patients who have undergone cholecystectomy as postcholecystectomy syndrome (PCS) occurs in $0.1-1.5 \%$ of the population, and up to $70 \%$ experience intrahepatic cholestasis [17]. Anxiety is another risk factor associated with increased perinatal morbidity and should be evaluated appropriately [18]. More frequent monitoring could relieve these symptoms and support the need for increased surveillance.

\section{Acknowledgements}

Thank you to Doctor David M. Greenspan, MD for his mentorship, guidance, and his passion for obstetrics that inspired this collaboration. Thank you to Doctor Lourdes Gonazlez, MD for your support and encouragement to complete this review.

\section{Consent Statement}

Written informed consent was obtained from the patient for publication of this article.

\section{Conflict of Interest}

Authors declare no conflict of interest.

\section{Data Availability}

All relevant data are within the paper and its Supporting Information files.

\section{References}

1. Media BK-K (2021) Publications \& Guidelines | SMFM.org - The society for maternal-fetal medicine.

2. Pusl T, Beuers $U$ (2007) Intrahepatic cholestasis of pregnancy. Orphanet Journal of Rare Diseases 2: 26.

3. Weizmann Institute of Science (2021) ABCB11 Gene (Protein Coding). Gene Cards. The Human Gene Database website. 
4. Pillarisetty LS, Sharma A (2021) Pregnancy intrahepatic cholestasis. Stat Peals.

5. Wang $H$, Yan Z, Dong M, Zhu X, Wang H, et al. (2012) Alteration in placental expression of bile acids transporters OATP1A2, OATPIB1, OATP1B3 in intrahepatic cholestasis of pregnancy. Arch Gynecol Obstet 285: 1535-1540.

6. Williamson C, Miragoli M, Sheikh Abdul Kadir S, AbuHayyeh S, Papacleovoulou G, et al. (2011) Bile acid signaling in fetal tissues: Implications for intrahepatic cholestasis of pregnancy. Dig Dis 29: 58-61.

7. Vasavan T, Deepak S, Jayawardane IA, Maristella L, Martin C, et a1. (2021) Fetal cardiac dysfunction in intrahepatic cholestasis of pregnancy is associated with elevated serum bile acid concentrations. J Hepatol 74: 1087-1096.

8. Williamson C, Gorelik I, Eaton BM, Lab M, Swiet M, et al. (2001) The bile acid taurocholate impairs rat cardiomyocyte function: A proposed mechanism for intrauterine fetal death in obstetric cholestasis. Clin Sci (Lond) 100: 363-369.

9. Egan N, Barbels A, Khashan A, Broadhurst CJ (2012) Reference standard for serum bile acids in pregnancy. BJOAG 119: 493-498.

10. Ovadia C, Seed PT, Sklavounos A (2019) Association of adverse perinatal outcomes of intrahepatic cholestasis of pregnancy with biochemical markers: Results of aggregate and individual patient data meta-analyses. The Lancet. 393: 899-909.
11. (2021) Cholestasis of pregnancy (ICP) - Symptoms, causes, and treatment. American Liver Foundation.

12. (2021) BAPS - Clinical: Bile acid profile, serum.

13. Lang T, McKeown G, Hunter A (2012) Biochemical investigation and clinical management of obstetric cholestasis (OC): How useful is bile acid quantification? Archives of Disease in Childhood - Fetal and Neonatal Edition 97: A50-A51

14. Manzotti C, Casazza G, Stimac T, Nikolova D, Gluud C (2019) Total serum bile acids or serum bile acid profile, or both, for the diagnosis of intrahepatic cholestasis of pregnancy. Cochrane Database Syst Rev.

15. Kirbas A, Biberoglu E, Daglar K, Iskender C, Erkaya S, et a1. (2014) Neutrophil-to-lymphocyte ratio as a diagnostic marker of intrahepatic cholestasis of pregnancy. Eur $\mathrm{J}$ Obstet Gynecol Reprod Biol 180: 12-15.

16. Williamson C, Geenes V (2014) Intrahepatic cholestasis of pregnancy. Obstet Gynecol 124: 120-133.

17. Barbier L, Souche R, Slim K, Ah-Soune P (2014) Long-term consequences of bile duct injury after cholecystectomy. Journal of Visceral Surgery 151: 269-279.

18. Orr ST, Reiter JP, Blazer DG, James SA (2007) Maternal prenatal pregnancy-related anxiety and spontaneous preterm birth in baltimore, maryland. Psychosom Med 69: 566-570. 